\title{
L'Apport de la typographie et de la musique à la poésie française du début du seizième siècle*
}

\author{
GASTON ALLAIRE
}

Il est remarquable de voir comment, à certains moments de l'histoire d'une société, une quelconque invention ou innovation peut, par ricochet, avoir un impact considérable sur un moyen d'expression artistique. Par exemple, tout près de nous, la radio, la télévision, l'industrie du disque et de la bande magnétique, médias de masse qui à leurs débuts ouvrirent leurs ondes à la musique, ont par ricochet si on peut dire, donné naissance plus tard à toute une poésie populaire qui s'exprime par la voix de nos chansonniers. Et ici, on ne peut s'empêcher de faire un rapprochement avec l'essor considérable de la chanson polyphonique française, surtout la chanson dite parisienne, pendant la première moitié du seizième siècle. Dans aucune autre période de l'histoire de la musique et de la littérature françaises voit-on une telle symbiose des deux arts, si ce n'est dans la mélodie romantique française de la fin du dix-neuvième siècle.

Mais que vient faire la typographie dans tout cela? Peu de temps après l'invention de l'imprimerie par Gutenberg vers 1440, c'est-à-dire en 1501, 1503, 1504, etc., l'imprimeur vénitien Petrucci publia ses fameuses collections de musique polyphonique. Pour l'époque et du point de vue typographique, ce sont des éditions impeccables. Mais ce qu'elles ont dû coûter de soins et d'argent, car ce système graphique devait se faire en deux étapes; la première par l'impression des lignes de la portée, la deuxième par l'impression superposée du texte et des notes de musique, procédé qui dût être excessivement difficile à accomplir si on voulait que les notes soient bien situées sur les lignes et dans les espaces. ${ }^{1}$

Ce qui attire l'attention lorsque l'on compare les éditions de Petrucci et celles du fameux imprimeur parisien Pierre Attaingnant parues un quart de siècle plus tard, c'est une impression en une opération au lieu de deux. Apparemment vers 1525, le typographe Haultin, "Graveur, Fondeur et Imprimeur à Paris," dont Attaingnant fut un des clients, eut

* Cet article, sans l'appendice musical, a fait l'objet d'une communication par une étudiante graduée du Département de Musique, Mlle Monique Pitre, à la réunion des sociétés savantes, i Fredericton, en mai 1977. 


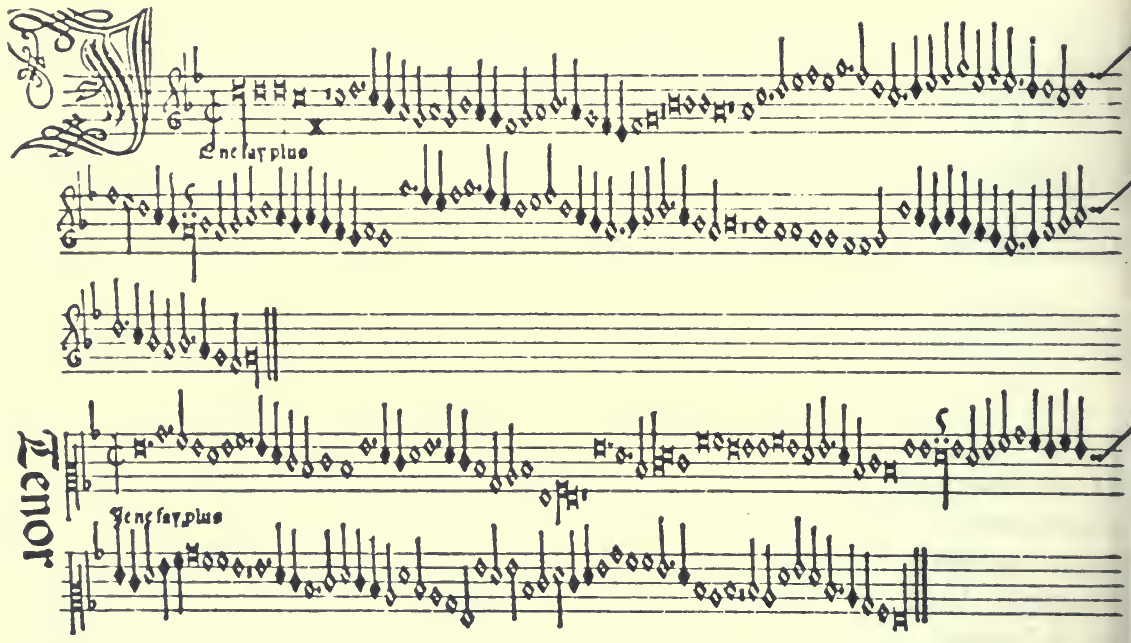

PETRUCCI - Harmonice musices odhecaton A - Venise, 1504, fol. $10^{\mathrm{V}}$

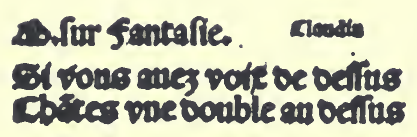

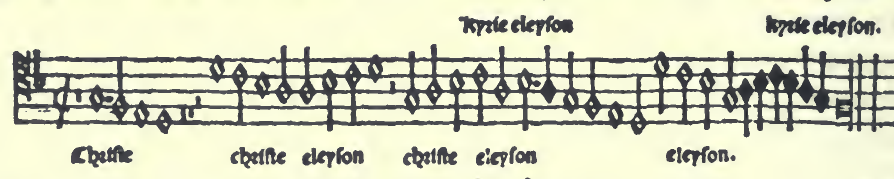

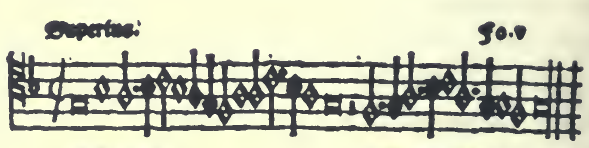

wric cievion

\section{troik clepion.}


l'idée de fondre une série de caractères qui représentaient les diverses valeurs des notes et des silences, mais celles-ci déjà placées sur les cinq lignes ou dans les espaces d'une courte portée. ${ }^{2}$ Ces caractères étaient placés sur un composteur exactement comme les caractères des lettres de l'alphabet, ce qui permettait une impression en une opération et représentait ainsi une économie de main-d'oeuvre considérable par rapport à l'impression en deux opérations de Petrucci.

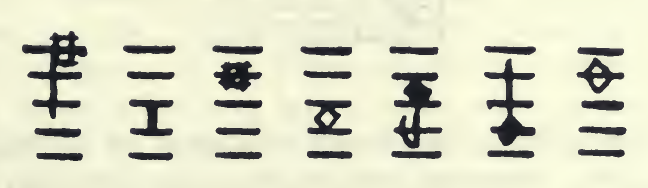

Exemple no 1

Il est vrai qu'avec cette nouvelle technique typographique on pouvait voir les joints entre les caractères, surtout dans les imprimés peu soignés, mais cela ne représentait pas un inconvénient majeur pour les musiciens, et l'énorme économie en salaires compensait largement ce léger défaut.

L'autre innovation apportée par Attaingnant fut la réduction du nombre des signes nécessaires pour réaliser une solmisation exacte (la solmisation est le nom donné au solfège d'avant 1600 ); cette nouvelle technique permettait de réaliser une économie de métal, d'encre, aussi bien que de main-d'oeuvre. Mais ceci supposait pour les chanteurs un changement assez radical dans la façon de lire leur musique, et c'est un changement qui semble s'être effectué dans un laps de temps très court, environ 25 ans.

La solmisation de la musique monodique pratiquée par les chanteurs n'était pas la même que celle de la musique polyphonique, c'est-à-dire la musique pour plusieurs voix de différentes étendues chantant simultanément. Or, d'après mes recherches, depuis le Moyen-Age on avait enseigné la solmisation par hexacordes aux garçons des maîtrises; la solmisation était nécessaire pour apprendre à chanter le plain-chant au nombreux offices religieux et les mélodies monodiques profanes aux cours princières. Parce que les voix d'hommes et d'enfants ou de femmes sont superposées dans le chant polyphonique, cette solmisation créait certains problèmes dont les détails techniques dépassent le cadre de cet article (Voir Appendice II); ces problèmes devaient être corrigés par l'utilisation d'un certain nombre de signes tels des fa-bémols aux armures, des armures apparemment incomplètes, et par un signe qui ressemble vaguement à notre dièze. 


\section{Exemple no 2}
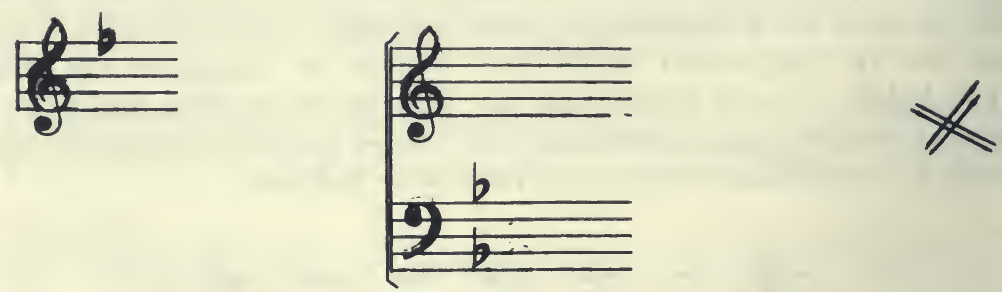

Mais pour le compositeur qui devait concevoir sa musique comme une toile couvrant deux hexacordes conjoints ou disjoints, il existait une solmisation par deux gammes, l'une dure et l'autre molle, qui ne nécessitait pas tous les signes de la solmisation par hexacordes. Tous les signes de la solmisation par hexacordes, nous les trouvons dans les imprimés de Petrucci $(1501,1503,1504)$ et même chez Antico aussi tard qu'en 1520. Mais soudain, dans les imprimés d'Attaingnant, à partir de 1527, il n'y a plus aucun des signes qu'on trouvait chez Petrucci moins d'un quart de siècle plus tôt. Que s'est-il passé au juste?

C'est ici que j'élabore l'hypothèse suivante pour tenter d'expliquer ce qui peut s'être passé. Au début du $16^{\mathrm{e}}$ siècle, la musique européenne était contrôlée par ce qu'on appellerait aujourd'hui la "mafia" francoflamande. Située au nord de Paris et s'étendant jusqu'à Anvers, cette région constituait un bassin de musiciens qui allaient oeuvrer dans toutes les cours importantes d'Europe. Paul-Henry Lang $^{3}$ a dénombré pas moins de 27 musiciens d'origine franco-flamande à Paris, 24 à Milan, 15 à Ferrare, 5 à Modène, 5 à Florence, 107 à Rome, 17 à Naples, 13 à Venise, 15 à Vienne, 10 à Munich, 9 à Dresden, 6 à Berlin, 47 à Madrid, et 10 à Tolède.

Il est intéressant de noter que Pierre Attaingnant était originaire de cette région, plus particulièrement de Douai en Artois, et aussi que Claudin de Sermisy (v. 1490-1562), compositeur favori des publications d'Attaingnant, était lui aussi originaire de Picardie. Toujours d'après Heartz, Attaingnant aurait très probablement reçu un entraînement musical dans la chorale de l'église de Douai, car dans toute cette région l'enseignement de la musique était à l'honneur. Plusieurs diocèses des vieilles provinces du nord de la France offraient des bourses d'études aux petits chanteurs entraînés dans les maîtrises diocésaines pour aller étudier à Paris. C'est ainsi qu'Attaingnant se serait retrouvé au collège de Dainville à Paris vers l'âge de 14 ans. Que le Picard Attaingnant ait fait la connaissance du Picard Claudin de Sermisy, qui était clercmusicien à la Sainte-Chapelle du Palais en 1508, n'aurait rien de surpre- 
nant, les deux hommes étant d'ailleurs à peu près du même âge. De plus, Attaingnant ne serait pas resté longtemps au collège; il l'aurait quitté pour entrer comme apprenti chez un imprimeur, sa bourse étant prolongée pour toute la durée de l'apprentissage. ${ }^{4}$

Pendant la Renaissance les imprimeurs de musique étaient presque tous musiciens; on peut citer, entre autres, Gardane, Antico, Le Roy, etc. L'art musical était un art encore assez ésotérique et hermétique à cette époque; seuls des musiciens compétents pouvaient se hasarder à imprimer la musique avec succès. Par conséquent, on peut spéculer que sous l'influence d'Attaingnant et de plusieurs autres imprimeurs du temps, il fut convenu de généraliser la solmisation polyphonique par gammes en remplacement de la solmisation traditionnelle par hexacordes, ce qui permettait une économie appréciable de métal, d'encre et de maind'oeuvre. Ce changement semble avoir été un mouvement général presque simultané partout et peut avoir été encouragé par le fait que la musique, en Europe occidentale, était dominée par les compositeurs Franco-flamands éparpillés dans toutes les cours importantes, et que les imprimeurs connaissaient la musique.

Y a-t-il eu collusion entre le Picard-imprimeur Attaingnant et les Picards-chanteurs à la cour de François $1^{\text {er }}$ lorsque Attaingnant se lança dans la publication de la musique vers 1515-1525? Y a-t-il eu mot d'ordre passé dans toutes les cours où des Picards oeuvraient comme musiciens de délaisser la solmisation par hexacordes et d'enseigner la solmisation par gammes dure et molle pour la musique polyphonique? On serait porté à le croire lorsque l'on constate qu'Attaingnant a publié quelques 230 compositions de Claudin, le plus grand nombre par un même compositeur, 178 de Jannequin, un autre Picard, et 188 de Certon, probablement un Picard lui aussi, et le protégé de Claudin de Sermisy.

L'immense production d'Attaingnant entre 1525 et 1550 , porte à croire qu'il pouvait produire ses collections de chansons polyphoniques à un prix très inférieur à celui des collections qu'on publiait un quart de siècle plus tôt, et que cette production répondait à une très forte demande de la part des consommateurs. La "mafia" franco-flamande étant répandue à travers l'Europe, comme on l'a vu plus tôt, il n'est pas surprenant de trouver la chanson polyphonique française à la mode dans toutes les cours importantes d'Europe, où les collections de chansons d'Attaingnant semblent avoir connu une diffusion très large. Donc, de 1525 à 1558 , environ 175 titres de collections - surtout des collections de chansons polyphoniques françaises - sont sorties des presses d'Attaingnant; c'est ainsi que pendant un peu plus de 25 ans Attaingnant en publia plus de 200 , une production impressionnante qui représente quelques 80 nouvelles chansons par année. 
On situe les origines de la chanson polyphonique française au début du quinzième siècle, à la cour des ducs de Bourgogne. Sous l'impulsion des compositeurs néerlandais Dufay, Ockeghem, Obrecht, Binchois et autres, naquit un style de musique qui se répandit à travers l'Europe. Cette musique était d'un style contrapuntique qui évoluera assez rapidement vers des points d'imitation. C'est ce style par points d'imitation qu'on trouve dans la chanson franco-flamande de Josquin des Prés qui innove en exploitant les points d'imitation par paires de voix.

\section{Exemple no 3}

(Créquillon, Susato 1543)

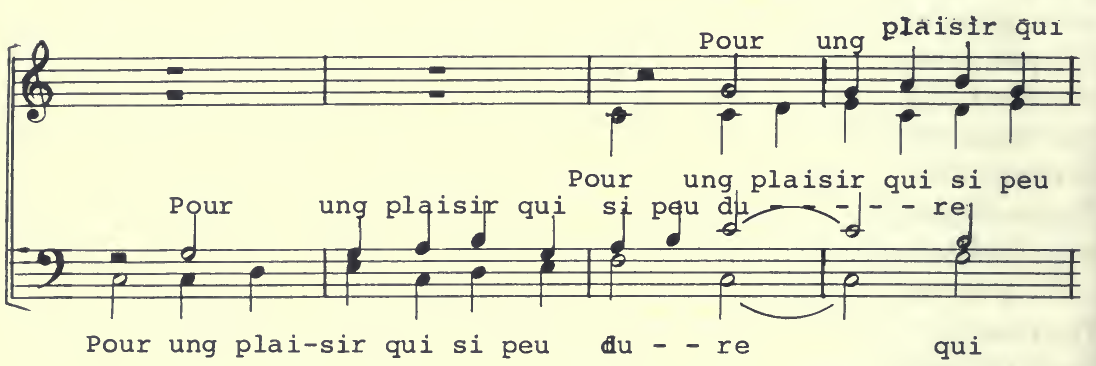

On ne connaît pas trop bien les relations de Josquin avec la cour de France, mais on sait qu'il était à Paris sous Louis XII, le prédécesseur de François $1^{\mathrm{er}}$, et qu'il y mourut en 1525. L'usage intensif de l'imitation par voix pairées qu'on trouve dans le style de Claudin de Sermisy suggère que celui-ci a étudié avec Josquin.

Mais vers 1525 un style nouveau apparaît dans la chanson polyphonique française, un style développé par Claudin de Sermisy qui sera exploité dans ce qu'on a convenu d'appeler la chanson polyphonique parisienne pour la distinguer de la chanson franco-flamande. Ce nouveau style se voulait plus simple et plus direct que le style franco-flamand, et par conséquent moins contrapuntique, organisé davantage par succession d'accords; il se prêtait mieux à la compréhension du texte, lequel était devenu avec le poète Clément Marot l'élément primordial des chansons.

Exemple no 4

(Claudin, Attaingnant $1536^{5}$ )

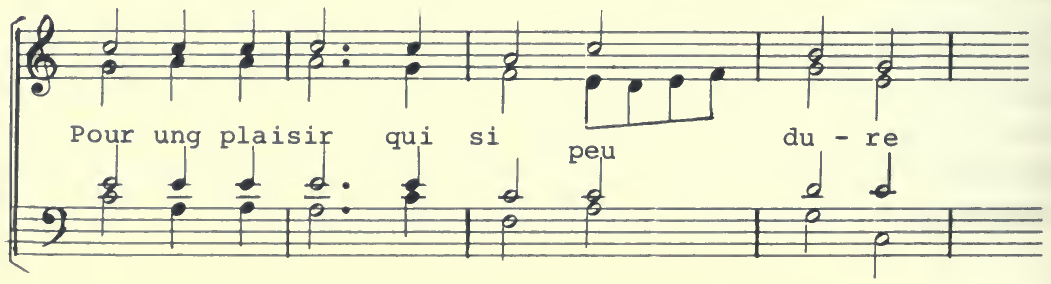


Alors que dans la chanson franco-flamande la mélodie principale n'était pas nécessairement chantée par la partie vocale la plus haute, dans la chanson parisienne c'est la voix la plus haute qui tend à primer avec sa mélodie. Que Claudin de Sermisy ait fait un effort conscient pour simplifier la musique et permettre ainsi au texte de ressortir plus clairement ne fait pas de doute. C'est alors que la poésie française acquit une popularité inconnue jusqu'alors, et que les poètes se multiplièrent. On compte pas moins de huit poètes connus dont les textes furent mis en musique par le seul Claudin de Sermisy: Le roi François $1^{\mathrm{er}}$, sa soeur Marguerite de Navarre, Mellin de Saint-Gelays, Octavien de SaintGelays, le cardinal François de Tournon, Claude Chappuys, Bonaventure des Périers, Antoine Héroet et Clément Marot, le protégé de la soeur du roi et le second grand poète français après Villon. Des 169 chansons connues de Claudin de Sermisy composées sur des oeuvres des poètes ci-dessus et d'autres, anonymes ceux-là, il y eut 442 éditions et rééditions entre 1528 et 1608 , surtout avant 1550 . Ceci nous donne une idée de la grande popularité de la chanson polyphonique parisienne en général et de la chanson de Claudin de Sermisy en particulier.

Si les compositeurs parisiens de la Renaissance furent fidèles à la tradition néerlandaise des Messes, Requiems, Motets, Passions et Lamentations, ils n'atteignirent un style vraiment national que dans la chanson polyphonique. De fait, la chanson polyphonique est tellement caractéristique de la culture française de la Renaissance qu'on peut même aller jusqu'à dire que sans elle on aurait peine à parler de Renaissance française en musique. D'ailleurs le style direct, de contrepoint simplifié, d'imitation par paires de voix et de sections homorythmiques (monodie accompagnée), de la chanson parisienne va s'infiltrer dans le style du Motet et de la Messe polyphonique parisienne qui devient plus courte, moins lourde et moins compliquée que sa contre-partie franco-flamande. La chanson polyphonique française a été un facteur déterminant dans l'évolution de la musique de cette époque puisqu'elle donna naissance à la canzona instrumentale italienne, laquelle évolua par la suite vers la sonate baroque.

Quant à la poésie, elle servit d'inspiration directe à la musique. Les poètes conçurent leurs poèmes en fonction de l'adaptation musicale, comme le suggère le mot "chanson" qu'ils utilisèrent souvent comme titre. Ici le mot devint la raison d'être du son, et non pas l'esclave ou le maître de la musique comme ce fut le cas à d'autres moments de l'histoire de la musique. Parce que les poètes étaient musiciens et que les musiciens étaient poètes, la coopération entre les arts soeurs fut intense et fructueuse. Dans "Quintil Horatian," cité par H. Chamard, Bartholemy Aneau affirme que le poète Mellin de Saint-Gelays (14911558) pouvait mieux que tout autre écrire des poèmes qu'il mettait en musique, les chantant et les jouant lui-même. Les textes poétiques de 
Saint-Gelays furent mis en musique par Claudin de Sermisy, Arcadelt, Belin, Cadéac, Delatre, Le Heurteur, Maillard, Sandrin, et d'autres, ce qui démontre leur popularité. Clément Marot a laissé au moins un air de musique dont il est certain qu'il est de lui. L'exemple de la collaboration entre la musique et la poésie venait de haut, des rois eux-mêmes. François $1^{\text {er }}$ était un poète de quelque distinction et peut-être même un peu musicien; Heartz suggère que la chanson polyphonique dont le compositeur est "Françoys" et qui fut publiée par Attaingnant aurait été composée par le roi lui-même. Au moins cinq de ses poèmes furent mis en musique par Jannequin, Claudin de Sermisy et d'autres compositeurs du seizième siècle: "Dictes sans peur," "J'ay le désir content," "Je n'ose être content," "Las que crains-tu amy," et "Voulant amour."

Mais, le poète le plus populaire de cette première moitié du seizième siècle est bien Clément Marot; environ 125 de ses poèmes furent mis en musique, dont 26 par Claudin de Sermisy et 18 par Clément Jannequin. Son épigramme paillarde "Martin menoit son pourceau au marché" fut mise en musique polyphonique par au moins cinq compositeurs, et cette musique apparaît dans au moins neuf éditions différentes. La version de Jannequin fut assez populaire pour faire l'objet de quatre éditions, sans compter les arrangements en tablature de luth, et la canzona pour instrument à clavier qu'en fit Andréa Gabrieli. Avec Marot le texte littéraire devint plus direct et même familier; les strophes étaient courtes et respectaient généralement l'alternance des rimes masculines et féminines. Plus tard Ronsard continuera dans la même voie et s'efforcera même de concevoir ses textes en fonction de l'adaptation musicale Il y avait chez les poètes d'alors le souci de voir la musique se marier avec la poésie, ainsi que le font voir les vers suivants de Ronsard:

\footnotetext{
Premier j'ay écrit la façon

D'accorder le luth aux odes.

Viens à moy, mon luth, que j'accorde

Une ode, pour la fredonner

Dessus la mieux parlante corde

Que Phoebus t'ait voulu donner....6
}

Si Marot et Ronsard, en s'efforçant dans leur poésie de se rapprocher de l'idéal musical, ont produit le genre de poèmes que les compositeurs du seizième siècle ont aimé mettre en musique, ceux-ci, par ailleurs, n'ont pas peu contribué à faire connaître l'oeuvre des poètes, si on en juge par les très nombreuses compositions et les très nombreuses rééditions qu'ont connues les chansons de Marot et de Ronsard. Selon l'expression moderne, on peut même dire que l'imprimerie musicale a contribué à lancer Marot et Ronsard. Examinons, par exemple, la biblio- 
graphie musicale de la chanson D'ou vient cela, belle, je vous supply, sur un texte de Marot dans l'Appendice I. Une telle bibliographie a pu faire dire à Jean Rollin:

\begin{abstract}
Voilà les sources auxquelles nous avons puisé pour trouver d'une part, les textes littéraires, d'autre part les textes musicaux des "Chansons". Rien que par cette revue rapide, on peut déjà apprécier le caractère étendu et inattendu de la documentation musicale et préjuger que notre poète a pu connaître grâce aux musiciens de son temps. Portés sur les ailes de la musique, les vers de Marot volent de Paris à Lyon, d'Anvers à Venise, de Londres à Séville. ... . Les catholiques chantent des messes (Messes-parodies à la mode au seizième siècle) bâties sur les mélodies devenues populaires, des chansons du poète; les Réformés entonnent des chants spirituels, puis des psaumes, sur les mêmes airs. ...?
\end{abstract}

Ces collections de chansons publiées par Attaingnant ont ceci de particulier qu'elles sont de véritables anthologies dans lesquelles on trouve des chansons de tous genres: spirituelles, amoureuses, anodines, à boire, à danser, paillardes ou franchement obscènes. Il y en avait pour toutes les occasions: les réunions amoureuses, les méditations spirituelles, le théâtre, les mascarades, les ginguettes (ancêtres de nos discothèques modernes?), et dans tous les genres depuis l'amour courtois jusqu'à l'érotisme le plus direct, de l'anecdote au potinage, de la profonde mélancolie à la folle gaieté, des considérations philosophiques aux descriptions du chant des oiseaux ou des batailles guerrières. Ces chansons étaient publiées dans quatre cahiers d'à peu près 6 pouces par $71 / 2$, un pour chaque partie vocale: Superius (cantus), Contratenor (altus), Tenor, et Bassus; il est fort probable que les chansons étaient chantées par seulement quatre musiciens - huit musiciens au maximum. On ne doit pas exclure la possibilité qu'une ou plusieurs parties vocales puissent avoir été jouées sur un ou plusieurs instruments mélodiques. Il est donc fort probable que les mêmes chanteurs qui chantaient la messe journalière de la cour, le matin, devaient chanter quelques chansons anodines et amoureuses pendant les repas de la cour, les chansons spirituelles lors de la prière du soir avant le coucher des enfants de la cour, et plus tard les chansons à boire, paillardes et obscènes dans les ginguettes ou tout autre lieu où le roi et les princes allaient passer une bonne partie de la nuit avec les femmes de petite vertu qui s'y trouvaient. Enfin, à l'occasion de représentations théâtrales, on devait faire appel aux mêmes chanteurs pour chanter avant, pendant et après les représentations.

Si Attaingnant fut l'imprimeur qui "lança" musicalement le poète Marot, ce sont les imprimeurs Le Roy \& Ballard (Paris), Nicolas du Chemin (Paris), et Jacques Moderne (Lyon), qui, dans la seconde moitié 
du seizième siècle, "lancèrent" le poète Ronsard avec leurs nombreuses ré-éditions des Amours du célèbre poète.

\section{Université de Moncton}

\section{Notes}

1 Daniel Heartz, Pierre Attaingnant Royal Printer of Music (University of California Press, 1969), pp. $107 \mathrm{ff}$.

2 Ibid., pp. 49, 50.

3 Paul-Henry Lang, Music in Western Civilization (New York, 1969), carte géographique "The Distribution of Flemish (and Franco-Flemish) Composers, During the Height of Their Dispersal c. 1470-1550."

4 Heartz,op. cit., p. 34.

5. H. Chamard, La Deffence et Illustration de la Langue Francoyse (Paris, 1948).

6 La $12^{\text {ème }}$ ode du premier livre publié en 1550 , et les premières lignes de la $3^{\text {ème }}$ ode du deuxième livre publié aussi en 1550 .

7 Jean Rollin, Les Chansons de Clément Marot (La Société Francaise de Musicologie, Paris, 1951), pp. 172, 173, et 45 .

\section{APPENDICE I}

MUSIQUE VOCALE:

\section{MUSIQUE MONODIQUE}

(Sans musique écrite, mais avec indication des timbres) ${ }^{\mathrm{a}}$

Anonyme S'ensuyvent plusieurs belles chansons, 1537.

Anonyme Plusieurs belles chansons, Lotrian-1543.

Beaulieu Chrestienne resjouissance, (Genève), 1546.

\section{MUSIQUE POLYPHONIQUE}

a) Chansons:

Sermisy

Sermisy

Sermisy

Sermisy

Créquillon

Anonyme
Créquillon

Chansons nouvelles (Paris) Attaingnant-s.d.

Chansons nouvelles (Paris) Attaingnant-1528.

Trente et sept chansons (Paris) Attaingnant-1532.

Livre 7e des chansons (Douai) Bogart-1633.

Le quatriesme livre (Anvers) Susato-1544.

Second livre des chansons (Louvain) Phalèse \& Rotaire1533, 1556, 1560.

Septiesme livre ou Livre 7e (Louvain) Phalèse-1570, $1576,1597,1601,1605,1608,1613,1622,1632$, 1633, 1636.

a Timbre: Motif mélodique tombé dans le domaine public et sur lequel les auteurs de cantilènes, parodies, chansons, vaudevilles, cantiques, noëls, etc. placent les paroles de leurs compositions. Larousse de la Musique (Paris, 1957). 
Anonyme Primo libro de le Canzoni Francese (Venise) Scotto1535.

Anonyme Il primo libro (Venise) Gardane-1543.

Anonyme Le treizi区́me livre (Anvers) Susato-1550.

b) Psaumes: (Texte transformé en Psaume $X$ par Marot lui-même, avec les paroles suivantes: D'ou vient cela Seigneur je te supply)

Goudimel Les Pseaumes mis en rime françoise (Genève) Jaqui$1565,1580$.

Goudimel Psalmen Davids (Hesborn) 1609.

Lassus Thrésor de musique (Cologne) Marceau-1594.

Sweelinck Les Psaumes de David (Amsterdam) 1604.

MUSIQUE INSTRUMENTALE:

Sermisy

Très brèves (Pour luth \& voix) (Paris) Attaingnant1529.

Anonyme Tablature des orgues (Paris) Attaingnant-1530.

Anonyme Nova. . cithara (Louvain) Phalèse-1568)

(Il est à remarquer que Pierre Attaingnant fut le premier imprimeur à publier des chansons polyphoniques sur des textes de Marot et leur arrangement pour instruments)

\section{APPENDICE II*}

Avant 1600, trois hexacordes de base étaient employés par les chanteurs pour la lecture de la musique monophonique (plain-chant appelé de nos jours chant grégorien, mélodies des troubadours et trouvères, toutes les mélodies populaires non harmonisées):

Hexacorde Durum:

Syllabes de solmisation:

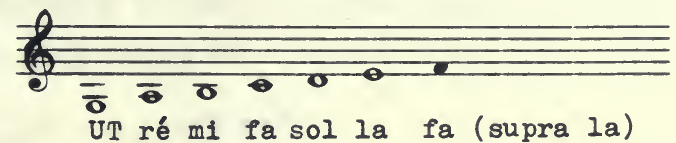

Hexacorde Naturale:

\section{Syllabes de solmisation:}

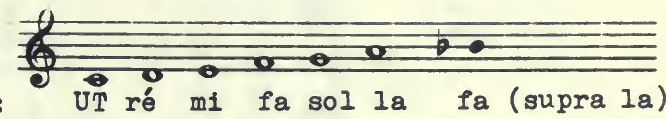

* Cet appendice est le résultat partiel d'une recherche à partir de mon traité The Theory of Hexachords, Solmization, and the Modal System: A Practical Application paru aux éditions de l'American Institute of Musicology en 1972 (MSD-25). Pour des données plus substantielles sur cette recherche, voir les articles suivants qui ont été acceptés pour publication: "Peculiar Signatures in $14^{\text {th }}, 15^{\text {th }}$, and $16^{\text {th }}$ Century Sources" dans Journal of CASUM/ACEUM, et "Les Enigmes de l'Antefana et du Double Hoquet de Machault: Une Tentative de Solution" dans Revue de Musicologie, Paris. 
Hexacorde Molle:

Syllabes de solmisation:

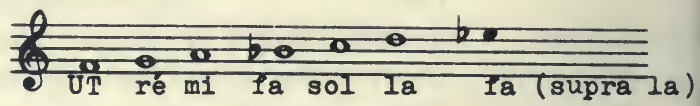

$\mathrm{Au}$ moyen de la transposition, l'hexacorde construit sur Si-bémol était aussi employé:

Syllabes de solmisation:

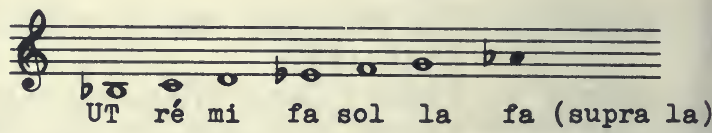

Pour simplifier les données du problème, nous ne nous en tiendrons qu'à la division arithmétique de l'octave (quarte sous quinte), laquelle est la division de presque tous les modes médiévaux en musique polyphonique:

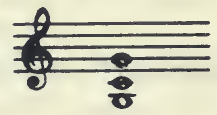

Dans cette division, un hexacorde central est toujours flanqué d'un autre hexacorde construit à la quarte supérieure et à la quinte inférieure, c'est-à-dire un hexacorde dans la direction de ceux du groupe des bémols:

Naturale - Durum - Naturale

Molle - Naturale - Molle

Hex. $\mathrm{Bb}-$ Molle - Hex. Bb

Si la solmisation par hexacordes convenait bien aux chants monophoniques, elle présentait un problème dans la musique polyphonique, car dans ce cas deux centres d'attraction pouvaient être établis en même temps, d'où des fausses relations d'octave, de quinte, ou d'unisson:

\section{Exemple a)}

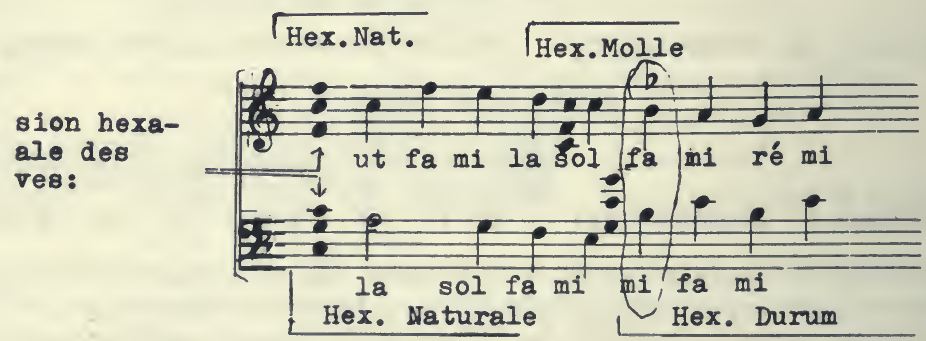

Cet inconvénient de la solmisation par hexacordes était évité par la neutralisation de l'hexacorde central, en l'occurence l'hexacorde 
Naturale, par la transformation de celui-ci en hexacorde Durum indiqué par le signe $\mathbb{W}$.

$$
\text { Exemple b) }
$$

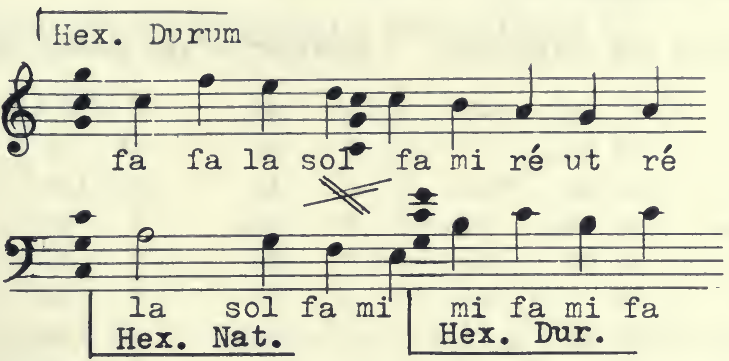

Dans les modes transposés en bémols (à la quarte supérieure ou à la quinte inférieure), le même problème se présentait:

\section{Exemple c)}

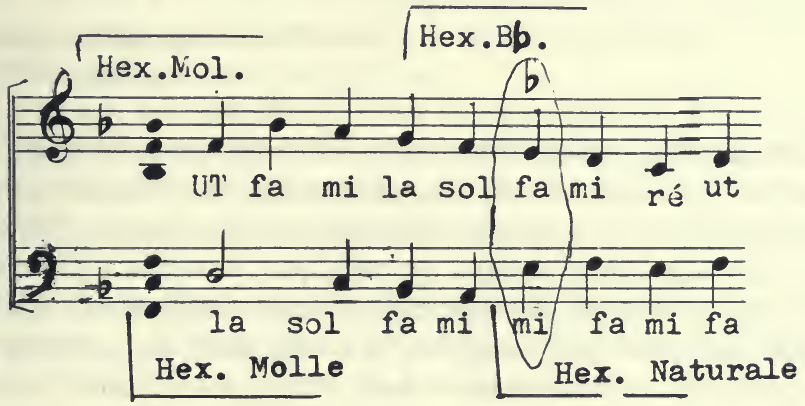

Ici c'est en enlevant le Si-bémol à l'armure de la voix supérieure que l'hexacorde central, le Molle, était neutralisé et transformé en hexacorde Naturale:

Exemple d)

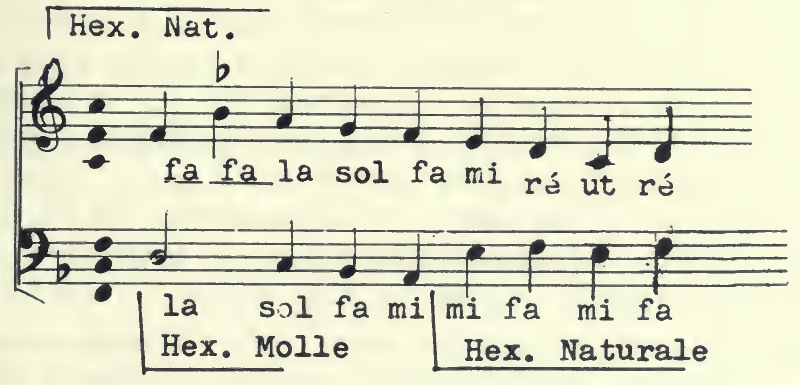


Mais après 1515 , on enseigna la solmisation polyphonique par gammes dure et molle, comme le fait voir le tableau comparatif suivant, dans lequel l'hexacorde central, le Naturale, est neutralisé par l'omission de ses syllabes motrices $u t$ et ré:

SOLMISATION PAR HEXACORDES

$\begin{array}{lll}\text { c } & \text { fa } & \text { UT } \\ \text { h } & \text { mi } & \\ \text { a } & \text { ré } & \text { la } \\ \text { G } & \text { UT } & \text { sol } \\ \text { F } & & \text { fa } \\ \text { E } & 1 a & \text { mi } \\ \text { D } & \text { sol } & \text { ré } \\ \text { C } & \text { fa } & \text { UT } \\ \text { Bh } & \text { mí } & \\ \text { A } & \text { ré } \\ \text { I } & \text { UT }\end{array}$

$\begin{array}{llc}\text { c } & \text { sol } & \text { UT } \\ \text { bb fa } & \\ \text { a } & \text { mi } & \text { la } \\ \text { G } & \text { ré } & \text { sol } \\ \text { F } & \text { UT } & \text { fa } \\ \text { E } & & \text { mi } \\ \text { D } & \text { la } & \text { ré } \\ \text { C } & \text { sol } & \text { UT } \\ \text { Bb fa } & \\ \text { A mi } & \\ \text { r ré } & \\ \text { F } & \text { UT }\end{array}$

SOLMISATION PAR GAMMBS DURE ET MOLLE

\begin{tabular}{|c|c|c|c|}
\hline c & $\rho a$ & c & sol \\
\hline 4 & $m 1$ & $b b$ & $f a$ \\
\hline a & $1 a$ & a & $1 a$ \\
\hline G & sol & G & sol \\
\hline F & $f a$ & $F$ & fa \\
\hline E & $1 a$ & $E$ & $\mathrm{mi}$ \\
\hline D & sol & $\mathrm{D}$ & $1 a$ \\
\hline $\mathrm{C}$ & $f a$ & $\mathrm{C}$ & sol \\
\hline Bh & $\mathrm{mi}$ & $\mathrm{Bb}$ & $f a$ \\
\hline A & ré & A & $\mathrm{mi}$ \\
\hline \multirow[t]{2}{*}{$\mathbf{r}$} & UT & $r$ & ré \\
\hline & & $F$ & UT \\
\hline \multicolumn{2}{|c|}{ Bogentantz, } & \multicolumn{2}{|c|}{$\frac{\text { Rudimenta }}{\text { musicae, }} 1515$ et 1535.} \\
\hline
\end{tabular}

Dans les modes transposés une fois en direction des bémols (à la quarte supérieure ou à la quinte inférieure) les tableaux ci-dessus doivent eux aussi être transposés à la quarte supérieure ou à la quinte inférieure, ce qui peut être fait dans un morceau de musique au moyen des clés mobiles.

Grâce à la solmisation par gammes dure et molle, après 1515, Attaingnant aurait imprimé les exemples a) et c), mais les chanteurs auraient utilisé les syllabes de solmisation des exemples b) et d). 